\title{
Rats assess costs and benefits according to an internal standard
}

\author{
Ruud van den Bos*, Johanneke van der Harst, Sietse Jonkman, \\ Mariska Schilders, Berry Spruijt \\ Ethology \& Welfare, Faculty of Veterinary Medicine, Utrecht University, Yalelaan 2, NL-3584 CM Utrecht, The Netherlands \\ Received 14 December 2005; received in revised form 20 March 2006; accepted 27 March 2006 \\ Available online 12 May 2006
}

\begin{abstract}
Variation in effort to obtain rewards is a fact of mammalian everyday life. In this study, we assess how rats scale variable costs and benefits. Different groups of rats were trained in a T-maze to discriminate a high (three or five sugar pellets) from a low reward (one sugar pellet) arm. Subsequently barriers were introduced at the high and low reward side such that the overall long-term pay-off of the high reward arm finally became lower than that of the low reward arm. The data show that under different regimes of costs (climbing barriers) and benefits (number of rewards) of the two arms rats appear to shift their behaviour towards the better side according to a constant relative cost-benefit ratio between the arms. Such a ratio allows them to deal with variation in the (physical appearance of) costs and benefits and choose the best long-term option.

(C) 2006 Elsevier B.V. All rights reserved.
\end{abstract}

Keywords: Rats; Iowa gambling task; Sugar pellets; T-maze; Barriers; Cost-benefit assessment

Variation in reward quality and magnitude is the rule rather than the exception for most organisms living under natural conditions. Therefore, both human and non-human animals trade off costs and benefits to optimise long-term behaviour [8,15]. As an example, it has been shown that humans choose the option with the best long-term pay-off in the Iowa Gambling Task, which contains moment-to-moment variation in costs (monetary losses) and benefits (monetary wins; 1). Effort to obtain rewards is another crucial factor in cost-benefit analyses. Several studies have been performed showing that animals assess costs and benefits of different options [6,7,11-13]. These studies have shown that choice behaviour of animals is determined by the number of food items that can be obtained and the barriers that need to be crossed. However, it has not been assessed thus far how cost-benefit ratios of different options are directly compared and how moment-to-moment variation in effort and reward affects long-term choice behaviour when multiple options exist. Given the efficiency of behaviour in an environment that contains variation $[8,15]$ it may be hypothesized that human and non-human animals scale variable costs and benefits according to a constant relative cost-benefit ratio. This allows them to deal with a

\footnotetext{
* Corresponding author. Tel.: +31 302534373 ; fax: +31 302539227

E-mail address: R.vandenbos@las.vet.uu.nl (R.van den Bos).
}

large number of costs and benefits, differing in actual (physical) appearance. We tested this in rats using a T-maze in which we introduced variation in rewards and effort.

For this experiment, $n=17$ Wistar rats (HsdCpb:WU, Harlan, The Netherlands) were used. All rats had already participated in another cost-benefit assessment experiment, which took place in a box different from the present experiment. They had been trained to discriminate arms differing in the proportion of sugar over quinine treated sugar pellets [2]. There was no evidence that this experiment had any effect on the present experiment. All experiments were approved by the Animal Ethical Committee of Utrecht University.

Three groups of rats were used that differed in age (A and B: 8-9 months; C: 15 months) and housing conditions. Rats of group A and B were housed socially $(n=2-3)$ under enriched conditions, i.e. a Macrolon type IV cage with a shelter, gnawing sticks and tissues. Rats in group $\mathrm{C}$ were housed socially $(n=3)$ under standard conditions (tissues), enriched conditions (gnawing stick, shelter, tissues) or in a Rathotel ( $n=6$ per cage, gnawing sticks, shelter, platform, ladders, tissues; Scanbur's Enriched Cage System; Scanbur AS, Denmark). Rats of group $C$ participated in an experiment on the effects of different housing conditions on standard laboratory tests. Of the seven rats reported here in group $\mathrm{C}, n=1$ was from a standard condition, $n=3$ from an enriched condition and $n=3$ from a Rathotel con- 
dition. Although proper statistics could not be run due to the low number of animals per housing condition, visual inspection of the data strongly suggested that no differences existed between the housing conditions on the values reported below.

All rats were housed in a temperature- and climate controlled room $\left(T=22 \pm 2{ }^{\circ} \mathrm{C} ; 40-60 \%\right.$ humidity) under a reversed $12 \mathrm{~h} / 12 \mathrm{~h}$ day-night cycle with lights off at 07:00 h. A radio provided background noise throughout the day. All testing was done during the dark phase between 10:00 and 16:00 h. Food was freely available, except during the testing period, when rats were give a limited amount of food keeping them to $95 \pm 5 \%$ of their free feeding weight; water was freely available throughout.

All experiments were run in a T-maze. This T-maze (cf. Ref. [12]) consisted of a start box $(l \times w \times h: 30 \mathrm{~cm} \times$ $25 \mathrm{~cm} \times 30 \mathrm{~cm})$, and two goal boxes $(30 \mathrm{~cm} \times 30 \mathrm{~cm} \times 60 \mathrm{~cm})$, connected by arms $(70 \mathrm{~cm} \times 30 \mathrm{~cm} \times 60 \mathrm{~cm})$. At the entrance of the arms two panels were positioned, around which the animals had to navigate and which blocked direct vision of the barriers. Animals were considered to have made a choice when they had climbed the first barrier. In most cases rats made a choice without inspecting the first barrier at either entrance.

Rats were trained to discriminate the arms of a T-maze by a procedure in which they were led through a series of forced choices, in which they sampled the arm's contents (three versus one or five versus one sugar pellets ( $45 \mathrm{mg}$; Bioserve Inc., Frenchtown, NJ, USA)), after which they could express their preference in a series of free choices. As rats had already been trained in a choice paradigm, they quickly learned to differentiate the arms. When they reliably chose the high reward arm more often than the low reward arm (criterion: $\geq 13 / 20$ choices at the high reward side), barriers, made of metal bars (diameter: $4 \mathrm{~mm}$; inter-bar distance: $2.5 \mathrm{~cm}$ ), were introduced across which rats had to climb to obtain rewards. The maximum number of barriers was three (inter-barrier distance: $20 \mathrm{~cm}$ ), with each barrier consisting of 16 bars maximally $(=40.0 \mathrm{~cm})$. The rats could not directly see the barriers when they left the start box. The total number of bars to be climbed at the 1 pellet side was fixed per 10 choices throughout testing. Within each block of 10 choices the actual barrier height per choice varied. The position of different pre-programmed barrier heights within each block differed across the successive blocks of 10 pre-programmed choices (cf. Ref. [1]). The number of bars to be climbed at the three or five pellet side was fixed for at least three choice sessions, i.e. until rats showed a stable choice. As for the 1 pellet side, within each block of 10 choices the actual barrier height per choice varied, and across successive blocks of 10 pre-programmed choices the position of the different pre-programmed barrier heights in each block differed (cf. Ref. [1]). Barrier height was successively increased. Tests ended when rats chose the low reward side more often than the high reward side. Only the data of the last session at each barrier height were used for analysis. Three groups (A-C) were tested that differed in the actual number of bars at the low reward side and number of pellets at the high reward side. Rats in group A and B differed in the number of pellets they could obtain at the high reward side (three and five pellets, respectively). Since the box had an upper limit of barriers $(n=3)$ and bars per barriers $(n=16)$ we decided to lower the average number of bars per trial (calculated per block of 10 pre-programmed trials) at the 1 pellet side in group B ( $2.0 \mathrm{bars} / \mathrm{trial} / \mathrm{pellet}) \mathrm{com}$ pared to group A (2.9 bars/trial/pellet). For rats in group C, i.e. the slightly older animals, that were given three pellets at the high reward side, we expected that they might experience some trouble to climb the barriers. We therefore decided to lower the average number of bars per trial (calculated per block of 10 preprogrammed trials) at the 1 pellet side to $1.5 \mathrm{bars} /$ trial/pellet.

Individual data for rats were plotted on an $x$ (ratio of bars at high reward versus low reward side) $-y$ (number of choices at the high reward side/10 trials of the last session) graph. For each rat a curve fitting procedure using a quadratic function $\left(y=a x^{2}+b x+c\right)$ was run, after which the ratio at the $x$-axis was calculated where $y=0$, i.e. when rats do not choose the high reward side anymore (see Fig. 1 panels $\mathrm{A}-\mathrm{C}$ for an example). $R$ square values indicated the goodness-of-fit per individual. The ratio-data, where $y=0$, were normalized for the amount of pellets at the high reward side, i.e. the costs that rats are willing to pay in terms of the number of bars they climb for one pellet at the high reward side relative to one pellet at the low reward side.

Statistics between groups were done using one-way analysis of variance (ANOVA) with post hoc testing as appropriate: $p \leq 0.05$ was considered significant, $0.05<p \leq 0.10$ a trend, and $p>0.10$ not significant (NS).

Fig. 1, panels A-C show the original data of groups A-C. As can be seen in these panels, rats slowly shifted towards the side where they could earn one pellet as the ratio of bars between the arms increased. For each individual a curve fitting was procedure was done to assess the point at which the animals would completely avoid the high reward arm $(y=0)$.

Fig. 1, panel D shows the normalized relative costs per pellet for the different test-groups. The data show that regardless of the actual number of bars at the low reward side, and regardless of the number of sugar pellets at the high reward side (three or five sugar pellets) rats will never visit the high reward side again, whenever the ratio of bars at the high reward side per pellet exceeds a value of $\sim 4.5$ relative to the low reward side (group A: $4.44 \pm 0.32$ (mean \pm S.E.M.; $n=5)$; B: $4.21 \pm 0.77(n=5)$; C: $4.87 \pm 0.59(n=7)$; one-way ANOVA: $F(2,14)=0.329$, NS). Closer inspection of the data showed that one rat in group $\mathrm{C}$ (rat 34) showed a rather low value. However when this rat was eliminated following an outlier test, statistical analysis still did not show an overall significant difference (one-way ANOVA: $F(2,13)=1.849, \mathrm{NS})$, although the ratio in group $\mathrm{C}(5.42 \pm 0.28$; $n=6)$ was slightly higher than in groups A and B.

These relative ratios were not related to a physical or upper limit of climbing bars. For instance, rats in group C $(21.93 \pm 2.68)$ tolerated, in absolute terms, significantly ( $p \leq 0.05$, Student-Newman-Keuls test) fewer bars per trial than rats in group A $(38.63 \pm 2.76)$ or B $(42.15 \pm 7.07$; one-way ANOVA: $F(2,14)=6.116, p \leq 0.012$ ), well below the maximum number of bars $(=48)$. Furthermore, the relative ratios were not related to any differences between rats after initial training. For instance, rats in group $\mathrm{C}(78.57 \pm 2.61 \%)$ chose the high reward side at criterion less often, but not significantly so (Student-Newman-Keuls-test, $p \leq 0.06$ ), than rats in group A $(92.00 \pm 3.74 \%)$ or B $(92.00 \pm 5.15 \%$; one-way ANOVA: 
$F(2,14)=4.744, p \leq 0.027$; see Fig. 1 , panels A-C). Finally, these relative ratios were not affected by differences in the reliability of the curve fitting procedures: $R$-square values, indicating the proportion of the variance explained by the equation did not differ between groups A, B and C (one-way ANOVA: $F(2,14)=1.795$, NS; Table 1).

The analysis of choice behaviour of rats in our three different experimental groups strongly suggests that rats use a constant ratio to decide between two options with different variable amounts of effort and rewards. This may aid the ani- mal in choosing the best long-term option under conditions of (moment-to-moment) variation in (physical appearance of) efforts and rewards at different locations. It is clear that more conditions should to be tested to make the point more strongly and to study which factors affect this ratio, such as age (compare the data of group $\mathrm{C}$ with those of groups A and B).

The question arises to what the underlying neuro-structure of this choice behaviour is. It has been shown that the anterior cingulate cortex [17] and ventral striatum [12] and their dopaminergic innervation $[7,14]$ may be critically involved in
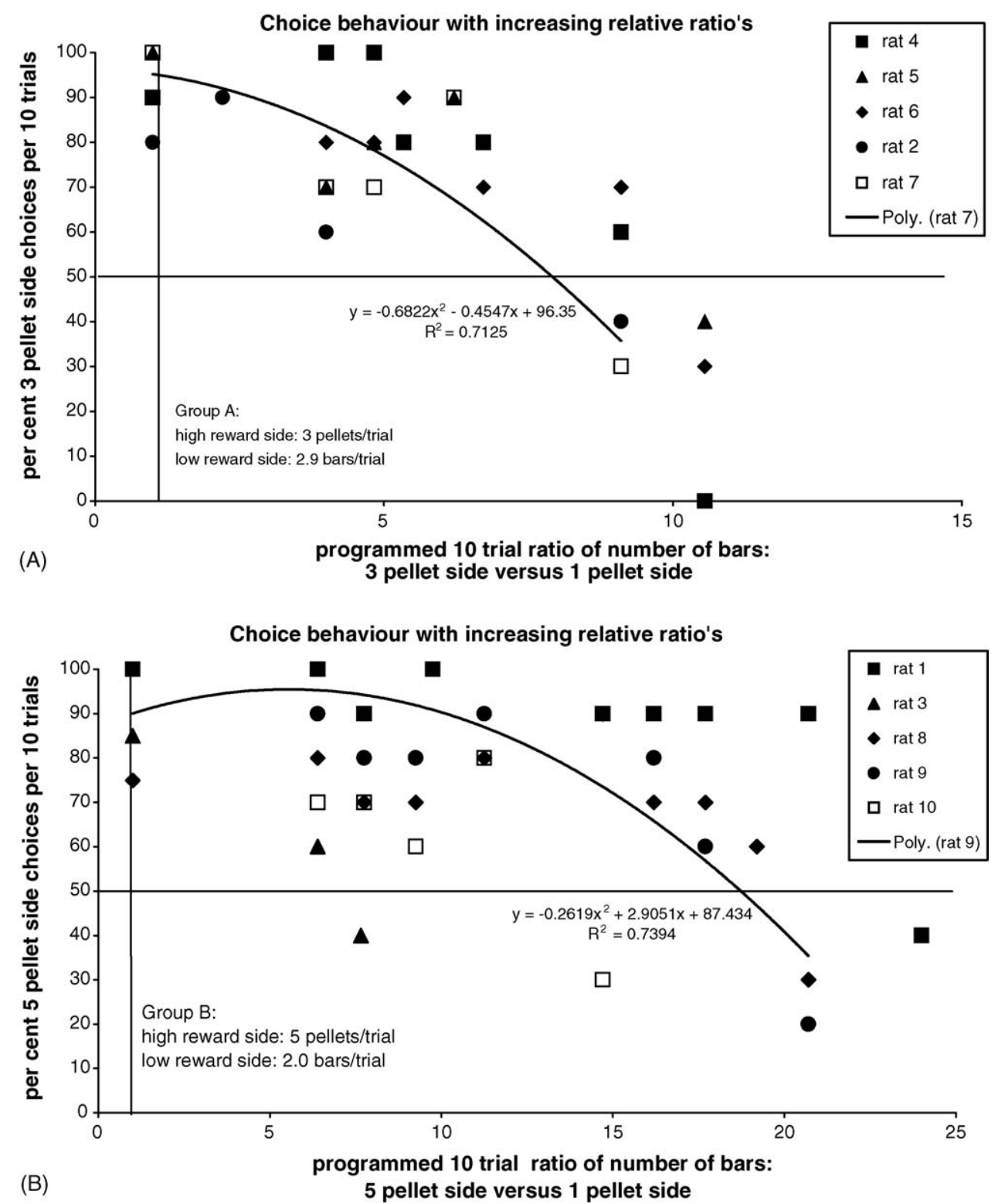

Fig. 1. (Panels A-C) Percent of choices per 10 trials for the high reward side under an increasing ratio of bars at the high reward versus the low reward side for individual rats in group A (panel A), B (panel B) and C (panel C). Symbols for individuals are the same for panel A-C on the one hand and panel D on the other. Panels A-C each show one example of a curve fitting procedure according to the equation: $y=a x^{2}+b x+c$; indicated are the parameters $(a, b$ and $c)$ and the proportion of variance explained by the equation ( $R$-square value). (Panel D) Normalized relative costs per pellet at the high reward side compared to the low reward side. Shown are individual data for rats in each group. The means per group are indicated by the horizontal bar. Pellets and bars are indicated per choice. For bars this is an average value of 10 pre-programmed choices at that side. Data for group $\mathrm{C}$ may serve as an example for calculating normalized costs. When on average the number of bars at the high reward side exceeds 21.93 per trial, rats do not choose the high reward side anymore. This leads to a ratio with respect to the low reward side of 14.62 for 3 pellets, or 4.87 per pellet, at the high reward side, which is the normalized ratio per pellet indicated as a group mean in the figure. 

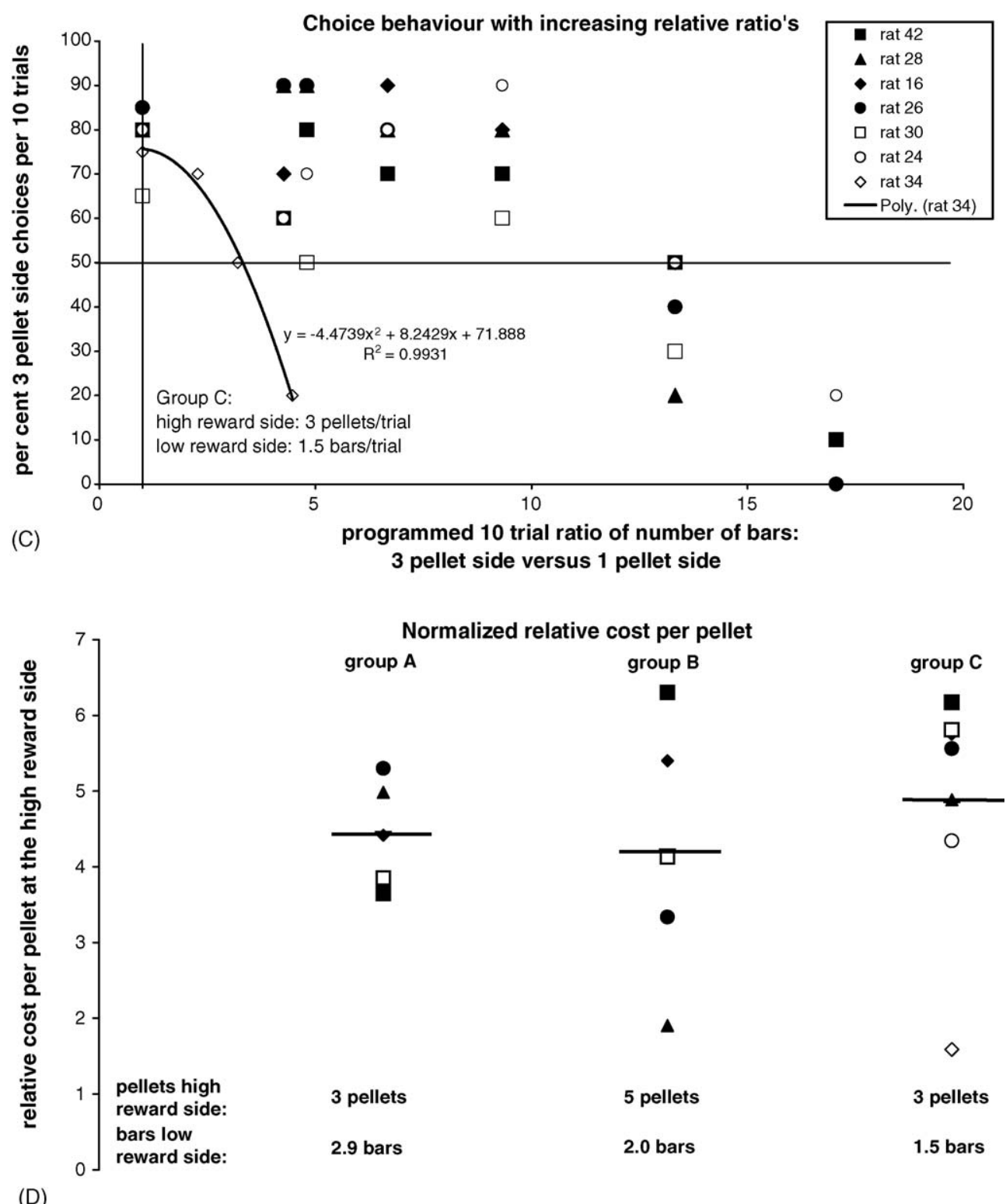

Fig. 1. (Continued).

Table 1

$R$-square values of curve fitting procedure

\begin{tabular}{llllll}
\hline Group A & \multicolumn{3}{c}{ Group B } & Group C \\
\hline Rat 4 & 0.92 & Rat 1 & 0.72 & Rat 42 & 0.89 \\
Rat 5 & 0.77 & Rat 3 & 1.00 & Rat 28 & 0.96 \\
Rat 6 & 0.82 & Rat 8 & 0.57 & Rat 16 & 0.94 \\
Rat 2 & $0.84^{\text {a }}$ & Rat 9 & 0.74 & Rat 26 & 1.00 \\
Rat 7 & 0.71 & Rat 10 & 0.72 & Rat 30 & 0.67 \\
& & & & Rat 24 & 0.76 \\
& & & & Rat 34 & 1.00
\end{tabular}

Mean \pm S.E.M. $\quad 0.81 \pm 0.03$

$0.75 \pm 0.07$

$0.89 \pm 0.05$

Unless otherwise indicated curve fitting is done according to the equation: $y=a x^{2}+b x+c$.

${ }^{a}$ Linear. choices encompassing barriers as effort. The anterior cingulate cortex is not involved in choice behaviour based on delays as effort, whereas the ventral striatum is [4], although data from recent studies tend to suggest otherwise with respect to (dopamine in) the ventral striatum $[9,16]$. Thus far it has been shown that (the prelimbic and infralimbic areas of) the medial prefrontal cortex $[4,5]$ and orbitofrontal cortex $[10,18]$ are sensitive to choices involving delays. Furthermore dopamine plays a role in choice behaviour based on delays, whereas serotonin is involved in choice behaviour based upon delays but not barriers [7]. This suggests a possible differentiation in cortical structures in regulating costs in terms of truly physical effort or costs in terms of waiting times, whereas at the subcortical level, i.e. the ventral striatum, no such difference exist (but see Refs. $[9,16])$, suggesting that this structure may be crucial in translating choices into sequences of goal-directed behaviour [3]. 
In conclusion, the data strongly suggest that rats scale the amount of costs to obtain rewards to a constant relative ratio, i.e. they seem to behave according to an internal constant factor allowing them to deal with variation in the (physical appearance of) costs and benefits and choose the best long-term option.

\section{Acknowledgement}

We wish to thank Annemarie Baars for technical assistance.

\section{References}

[1] Bechara A, Damasio AR, Damasio H, Anderson AW. Insensitivity to future consequences following damage to human prefrontal cortex. Cognition 1994;50:7-15.

[2] van den Bos R, Baars AM, Jonkman S, Spruijt B. Cost-benefit assessment in rats. Acta Neurobiol Exp 2003;63:S49.

[3] van den Bos R, Cools AR. Switching to cue-directed behavior: specific for ventral striatal dopamine but not ventral pallidum/substantia innominata GABA as revealed by a swimming test procedure in rats. Neuroscience 2003;118:1141-9.

[4] Cardinal RN, Pennicott DR, Sugathapala CL, Robbins TW, Everitt BJ. Impulsive choice induced in rats by lesions of the nucleus accumbens core. Science 2001;292:2499-501.

[5] Cardinal RN, Winstanley CA, Robbins TW, Everitt BJ. Limbic corticostriatal systems and delayed reinforcement. Ann N Y Acad Sci 2004;1021:33-50.

[6] Cousins MS, Atherton A, Turner L, Salamone JD. Nucleus accumbens dopamine depletions alter relative response allocation in a T-maze cot/benefit task. Behav Brain Res 1996;74:189-97.

[7] Denk F, Walton ME, Jennings KA, Sharp T, Rushworth MFS, Bannerman DM. Differential involvement of serotonin and dopamine systems in cost-benefit decisions about delay or effort. Psychopharmacology 2005;179:587-96.

[8] McFarland D. Animal economics. In: McFarland, editor. Animal Behaviour: Psychobiology, Ethology \& Evolution. Singapore: Longman Singapore Publishers Ltd.; 1993.
[9] Mingote S, Weber SM, Ishiwari K, Correa M, Salamone JD. Ratio and time requirements on operant schedules: effort-related effects of nucleus accumbens dopamine depletions. Eur J Neurosci 2005;21:1749_ 57.

[10] Mobini S, Body S, Ho MY, Bradshaw CM, Szabadi E, Deakin JFW, et al. Effects of lesions of the orbitofrontal cortex on sensitivity to delayed and probabilistic reinforcement. Psychopharmacology 2002;160: 290-8.

[11] Salamone JD, Steinpreis RE, McGullough LD, Smith P, Grebel D, Mahan K. Haloperidol and nucleus accumbens dopamine depletion suppress lever pressing for food but increase free food consumption in a novel food choice procedure. Psychopharmacology (Berlin) 1991;104:515-21.

[12] Salamone JD, Cousins MS, Bucher S. Anhedonia or anergia? Effects of haloperidol and nucleus accumbens dopamine depletion on instrumental response selection in a T-maze cost/benefit procedure. Behav Brain Res 1994;65:221-9.

[13] Salamone JD, Wisniecki A, Carlson BB, Correa M. Nucleus accumbens depletions make animals highly sensitive to high fixed ratio requirements but do not impair primary food reinforcement. Neuroscience 2001;105:863-70.

[14] Salamone JD, Correa M. Motivational views of reinforcement: implications for understanding the behavioral functions of nucleus accumbens dopamine. Behav Brain Res 2002;137:3-25.

[15] Spruijt BM, van den Bos R, Pijlman FTA. A concept of welfare based on reward evaluating mechanisms in the brain: anticipatory behaviour as an indicator for the state of reward systems. Appl Anim Behav Sci 2001;72:145-71.

[16] Wakabayashi KT, Fields HL, Nicola SM. Dissociation of the role of nucleus accumbens dopamine in responding to reward-predictive cues and waiting for reward. Behav Brain Res 2004;154:19-30.

[17] Walton ME, Bannerman DM, Alterescu K, Rushworth MFS. Functional specialization within medial frontal cortex of the anterior cingulate for evaluating effort-related decisions. J Neurosci 2003;23:6475-9.

[18] Winstanley CA, Theobald DEH, Cardinal RN, Robbins TW. Contrasting roles of basolateral amygdala and orbitofrontal cortex in impulsive choice. J Neurosci 2004;24:4718-22. 